\title{
Strategies for Adopting and Implementing SNOMED CT in Korea
}

\author{
Hyeoun-Ae Park ${ }^{1}$, Seung-Jong $\mathrm{Yu}^{2}$, Hyesil Jung ${ }^{3}$ \\ ${ }^{1}$ College of Nursing, Seoul National University, Seoul, Korea \\ ${ }^{2}$ InfoClinic Co. Ltd., Seoul, Korea \\ ${ }^{3}$ Office of Digital Medicine, Seoul National University Bundang Hospital, Seongnam, Korea
}

Objectives: The objective of this study was to introduce the Systematized Nomenclature of Medicine Clinical Terms (SNOMED CT), to describe use cases of SNOMED CT with the barriers and facilitators, and finally, to propose strategies for adopting and implementing SNOMED CT in Korea as a member of SNOMED International. Methods: We reviewed a collection of SNOMED CT documents, such as introductory materials, practical guides, technical specifications, and reference materials provided by SNOMED International and the literature on SNOMED CT published by researchers to identify use cases of SNOMED CT with barriers and facilitators. We also surveyed the attendees of SNOMED CT education and training series offered by the Korea Human Resource Development Institute for Health and Welfare to identify perceived barriers to adopting SNOMED CT in Korea. Results: We identified the barriers and facilitators to adopt SNOMED CT experienced by international terminology experts and prospective Korean users. They were related to governance and infrastructure, support services for use, education and training programs, use cases, and vendor capability to implement SNOMED CT. Based on these findings, we identified strategies for adopting and implementing SNOMED CT in Korea. They included the establishment of SNOMED CT management infrastructure, the development of SNOMED CT education and training programs for various user groups, the provision of support services for SNOMED CT use, and the development of SNOMED CT use cases. Conclusions: These strategies for the adoption and implementation of SNOMED CT need to be executed step by step.

Keywords: Systematized Nomenclature of Medicine, Terminology, Health Information Interoperability, Medical Informatics, Electronic Health Records

Submitted: December 31, 2020

Revised: January 22, 2021

Accepted: January 24, 2021

\section{Corresponding Author}

Hyesil Jung

Healthcare ICT Research Center, Office of eHealth Research and Businesses, Seoul National University Bundang Hospital, 82, Gumi-ro 173 Beon-gil, Bundang-gu, Seongnam 13620, Korea. Tel: +82-31-7878964,E-mail: 99526@snubh.org (https://orcid.org/0000-00028346-9343)

This is an Open Access article distributed under the terms of the Creative Commons Attribution Non-Commercial License (http://creativecommons.org/licenses/by$\mathrm{nc} / 4.0 /$ ) which permits unrestricted non-commercial use, distribution, and reproduction in any medium, provided the original work is properly cited.

(C) 2021 The Korean Society of Medical Informatics

\section{Introduction}

The ultimate goal of introducing healthcare information technology is to collect, share, and utilize the data generated in the field of healthcare. Although large amount of data are being generated in healthcare, they are not being utilized efficiently because of issues related to data sharing, such as lack of interoperability and data security problems.

Interoperability means the ability of various information systems, devices, and applications (systems) to access, exchange, integrate, and cooperatively use data in a coordinated manner, within and across organizational, regional, and national boundaries [1]. First, structural interoper- 
ability, which defines the type, syntax, and components of exchanged data, should be secured; the Health Level 7 (HL7) Clinical Document Architecture (CDA) and Fast Healthcare Interoperability Resources (FHIR) are used for this purpose. Next, the semantic interoperability should be secured to preserve the semantics of the exchanged data, and terminology standards, such as the International Classification of Diseases 10th Revision (ICD-10), SNOMED Clinical Terms (SNOMED CT), and Logical Observation Identifiers Names and Codes (LOINC) are used for this purpose. Finally, the processes and workflows related to data exchange should be defined. Terminology standards that enable users to express exchanged data, information, and knowledge clearly without ambiguity are the most important among these standards.

Health terminologies are classified into interface terminologies, which are used for the interactions between users and systems; reference terminologies that consist of concepts, relationships between concepts, and formal definitions; and classification systems, which are sets of mutually exclusive categories for aggregating data. Examples of interface terminologies are the local codes used by healthcare institutions such as VA Terminology Lexicon, and Mayo Clinic's Master Sheet Index. ICD-10 is an example of classification systems. The ideal utilization of health terminologies is to record the data collected by healthcare workers at the point of care using natural languages or interface terminologies into Electronic Health Records (EHRs), to map and store the EHR contents to the reference terminology and to use the data stored in the reference terminology for various purposes, including research, insurance claims, reports, and statistics. This requires mapping of the interface terminologies and classification systems to the reference terminologies.

SNOMED CT is one of health terminologies that satisfies the criteria for reference terminologies, such as concept orientation, formal definitions, poly-hierarchy, and multiple granularities [2]. SNOMED CT is the most widely used clinical terminology. It is used in more than 80 countries and more than 5,000 individuals and organizations around the world. Korea has joined the global effort to develop, maintain, and enable the use of SNOMED CT to achieve semantic interoperability of healthcare data, becoming the 39th member of SNOMED International in August 2020.

This review paper introduces SNOMED CT, describes use cases of SNOMED CT with the barriers and facilitators, and finally proposes strategies for adopting and implementing SNOMED CT in Korea as a member of SNOMED International.

\section{Overview of SNOMED CT}

SNOMED CT is a clinical terminology, which combined the SNOMED RT developed by the College of American Pathologists with the Clinical Terms Version 3 (formerly known as Read Codes) developed by the NHS of UK. SNOMED $\mathrm{CT}$ is the world's most comprehensive multilingual clinical terminology. SNOMED CT International Edition published on July 31,2020 contains 354,384 clinical concepts, 1,493,123 descriptions of the concepts, and 3,041,518 relationships between the concepts [3]. Currently, the SNOMED CT contents and licenses are maintained by SNOMED International with 39 countries as members of the organization.

SNOMED CT contents consist of three core components, namely, concepts, descriptions, and relationships. Concepts represent unique clinical ideas. A concept has a unique numerical concept identifier and at least one fully specified name (FSN). Concepts are organized in hierarchies from general concepts to more detailed concepts. Descriptions are readable textual expressions associated with concepts. A single concept may have multiple descriptions, including the FSN, preferred term, and synonyms. Relationships link one concept to another concept. The relationships include subtype (IS-A) relationships, which express relationships between concepts within a top-level hierarchy, and attribute relationships, which express associations between two concepts from two different top-level hierarchies. Each attribute relationship has a domain concept (the source of the relationship), an attribute (the type of the relationship), and a range concept (the destination of the relationship). The SNOMED CT relationships are used to make formal definitions of the concepts and to perform queries. The subtype (IS-A) relationship can be used to perform queries using more abstract (less detailed) concept that includes more specific (or more detailed) concepts. For example, when a query is executed to find "disorder of foot," more detailed concepts such as "cellulitis of foot," "diabetic foot," and "aseptic necrosis of talus" are included in the query result. The attribute relationship can be used to perform queries using the value of an attribute. For example, when a query is executed to find concepts with an "associated morphology" relationship with the value "benign neoplasm," the concepts such as "benign tumor of kidney," "benign neoplasm of bladder," and "benign tumor of lung" are included in the query result [4].

In addition to core components, there are SNOMED CT reference sets, a standard format for maintaining and distributing sets of references to SNOMED CT components to support various user requirements. The reference sets 
include subsets with frequently used concepts and descriptions, preferred descriptions in a specific language, and maps defining the relationships between SNOMED CT and other code systems, such as ICD-10 and LOINC.

All SNOMED CT concepts are subtypes of a root concept called "SNOMED CT Concept." There are 19 top-level concepts under the root concept. They include body structure, clinical finding, environment or geographical location, event, observable entity, organism, pharmaceutical/biologic product, physical force, physical object, procedure, qualifier value, record artifact, SNOMED CT Model Component, situation with explicit context, social context, special concept, specimen, staging and scales, and substance. The concepts descend from these 19 top-level concepts through at least one sequence of subtype (IS-A) relationships.

\section{Use Cases of SNOMED CT}

Figure 1 presents the component parts in which SNOMED CT is implemented in an EHR system and external systems. In the SNOMED CT documentation, the user interface, data storage, and external data communication are considered collectively to be part of clinical record implementation. Other types of implementation include linking to knowledge resources and aggregation and analysis. Terminology and reference data, such as SNOMED CT or ICD-10, are distinguished from clinical records in the clinical data storage. The analysis data store, in which some copies of the collection of clinical data records are held is separate from clinical data storage and terminology/other reference data resources to support data analysis and reporting [5].

Representative use cases of SNOMED CT according to the component parts are introduced here.
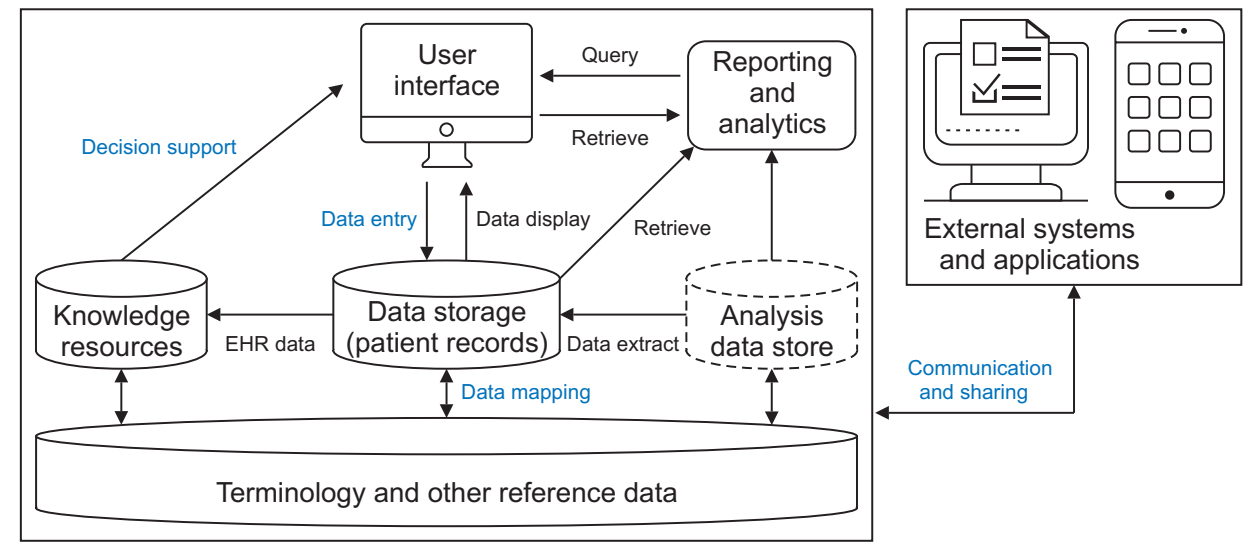

Figure 1. The component parts in which the SNOMED Clinical Terms (SNOMED CT) is implemented in an Electronic Health Record (EHR) system and external systems. 


\section{Decision Support}

The University of Nebraska Medical Center developed a statistical model to assess the risk for Alzheimer's disease using the patients' clinical data encoded with SNOMED CT and LOINC [9].

The Vanderbilt University Medical Center developed a decision support system for the identification of adverse effects and complications related to implantable cardiac electronic devices from patient notes using a natural language-processing technology based on the subtype (IS-A) and attribute relationships between SNOMED CT concepts [10].

\section{Communication and Sharing}

The NHS of the UK developed an electronic prescription system, in which a general practitioner (GP) uses SNOMED CT to prescribe a medicine and enter relevant clinical information. The GP then transmits the prescription to a pharmacy appointed by the patients and makes an insurance claim for the provided service [11]. The GP is required to record the detailed information of the patient in the summary care records (SCRs) using SNOMED CT. Healthcare workers can access SCRs to identify and share a patient's clinical information in an emergency [12].

\section{Public Report}

The Australian Digital Health Agency implemented SNOMED CT in the infectious disease reporting system for tracking and dealing with outbreaks of community-associated infections, including Escherichia coli infections in public swimming pools, rotavirus infections at childcare centers, legionella infections, salmonella food poisoning incidents, and methicillin-resistant Staphylococcus aureus infections.

The Canadian public health surveillance system employs SNOMED CT to record clinical information, such as diseases, antigens, antibodies, symptoms, and their causes; to track the vaccination history of citizens; to allow the early detection of infectious diseases and lockdown [13].

\section{Full Use of SNOMED CT in EHR System}

Hospital Italiano in Argentina introduced an EHR system with 1.5 million SNOMED CT concepts and 7 million descriptions, which it has used since 2003. The hospital manages a terminology server for maintaining SNOMED CT concepts, descriptions, and relationships. The terminology server automatically encodes the data entered by healthcare workers with SNOMED CT. The SNOMED CT-based EHR system developed by Hospital Italiano is used in more than 250 healthcare institutions in South American countries, such as Argentina, Uruguay, and Chile [14].

\section{Barriers and Facilitators for Adopting and Implementing SNOMED CT}

\section{Barriers}

We identified barriers to adopting and implementing SNOMED CT by reviewing two international survey reports, one survey of health terminology experts from five countries where SNOMED CT is implemented [15] and the other survey of experts from member countries and non-member countries of the EU SNOMED International [16]. We also surveyed 60 attendees of the SNOMED CT education and training series offered by the Korea Human Resource Development Institute for Health and Welfare to identify perceived barriers to adopting SNOMED CT in Korea. Most of them were health information managers and researchers belonging to healthcare institutions, and they were interested in mapping between SNOMED CT and local codes to use SNOMED CT in clinical practice and research. The identified barriers are as follows.

\section{1) Low awareness of the need for SNOMED CT}

There is a low level of understanding of the potential consequences or benefits of using SNOMED CT by users. It may be due to the low availability of best practices or good examples of SNOMED CT use in clinical settings. In addition, healthcare institutions have low awareness of the actual return on investment by adoption and utilization of SNOMED CT.

\section{2) Absence of SNOMED CT governance and infrastructure}

There is no or lack of governance and infrastructure for SNOMED CT management, such as licensing; language extension management; reference set, mapping, and translation, authoring tools, education, and training; engagement with stakeholders, and development of an implementation guide.

3) Insufficient SNOMED CT training and education programs The education and training programs are insufficient to address the current deficiency of SNOMED CT expertise and competencies of users. Various training and education programs (e.g., mapping, implementing, authoring, and data analytics) are required for various levels and roles of SNOMED CT users. 
4) Lack of support service for SNOMED CT use

SNOMED CT is difficult to understand because of its complex structure, and it is difficult to manage because of its large scale in clinical settings. Nonetheless, there is not enough guidance or support to promote the use of SNOMED CT, such as browsing, searching, navigating, authoring, translation, mapping, reference set development, and implementation. In particular, the guidance documentation and tools for implementing SNOMED CT step by step and addressing the relevant problems are insufficient.

5) Lack of vendor capability to implement, search, and display SNOMED CT

Vendors lack knowledge and skills to implement SNOMED CT in EHR systems, such as terminology management and data management. In detail, there is lack of the optimized search capability and usability in a clinical setting. In addition, they lack capability to leverage and use SNOMED CT for reference terminology. There is not enough data entry capability that takes advantage of SNOMED CT.

\section{Facilitators}

To overcome these barriers, the health terminology experts suggested an incremental step-wise plan for introducing and adopting SNOMED CT. First of all, it is important for the government to state a clear direction on the use of health terminology. It is necessary to increase awareness of SNOMED CT in individuals and healthcare institutions by developing use cases. They also suggested SNOMED CT governance to manage SNOMED CT licensing, supporting tools, and services to improve the usability of SNOMED CT by translation, mapping, and developing reference sets for specific diseases and categories and to share the experiences of users. In addition, professional training and education on the use of SNOMED CT are needed.

\section{Strategies for Adopting and Implementing SNOMED CT}

1. Establishment of SNOMED CT Management Infrastructure First, it is necessary to establish an infrastructure and governance, which would function as the National Release Center of SNOMED CT in Korea. The Data Standard Utilization Center at the Korea Health Information Service will play this role. This body must raise the understanding of SNOMED CT and promote the use of SNOMED CT in Korea. A Korean SNOMED CT browser must be developed for Korean users. It is important to provide tools for developing, updating, and managing a Korean extension of SNOMED CT as well as maps between local codes or other codes and SNOMED $\mathrm{CT}$, and reference sets. It is also necessary to operate a Help Desk for users to ask questions about SNOMED CT and a user community to share experiences and information among users.

\section{SNOMED CT Training and Education Programs}

Training and education programs must be developed and implemented for various levels and roles of SNOMED CT users. These would include a basic course that introduces beginners to a broad range of SNOMED CT related topics, including the why, what, and how to use SNOMED CT; an implementation course to provide an in-depth understanding of the SNOMED CT specific knowledge and skills required to implement a SNOMED CT enabled system; an authoring course to teach the knowledge and skills needed to manage SNOMED CT core components; a data analysis course to provide an understanding of how SNOMED CT can be used to support clinical data analytics requirements; and a software developer course to teach users enough about SNOMED CT to enable them to develop software applications that leverage its capabilities.

\section{Support Service for SNOMED CT Use \\ 1) Translation and Korean extension}

Korean translation and extension of SNOMED CT are necessary to increase the usability of SNOMED CT by Korean users and their acceptance and to improve international interoperability. A Korean extension may include the Korean terms in the KOrean Standard Terminology Of Medicine (KOSTOM) developed by the Ministry of Health and those in the local codes used by healthcare institutions as descriptions.

\section{2) Mapping}

Maps between the Korean classification of diseases, health insurance claim codes, disease registry, health information exchange forms and data items, local codes, and SNOMED CT must be developed along with mapping guidelines for the successful adoption and promotion of SNOMED CT.

\section{3) Reference sets}

The scale of SNOMED CT contents makes it challenging to implement. Thus, small reference sets can be developed for specific diseases or specialties. For example, reference sets may be developed for data items collected in the $\mathrm{Na}$ tional Cancer Registration and Statistics Program or the 
COVID-19 reporting system. Clear guidance and tools for developing and maintaining reference sets are also needed.

\section{SNOMED CT Use Cases}

1) Use of SNOMED CT in EHR

In EHR systems, SNOMED CT can be used as a reference terminology for data communication with external systems; as a reference terminology for data integration; as an indexing system for data retrieval; as a code system for clinical data storage; as an interface terminology for data entry; and as an extensible foundation for representing clinical data through post-coordination, for knowledge linkage, and for simple aggregation and analysis of data [5].

\section{2) Use of SNOMED CT in national health IT initiatives}

(1) Certification of EMRs systems: The program for the certification of Electronic Medical Records (EMRs) systems recommends the use of standard terminologies, even though it is not mandatory. For example, family medical history, medical history, and social history will be mapped to SNOMED CT as part of the EMRs certification program.

(2) Healthcare information exchange: In the current healthcare information exchange program, HL7 CDA is used to exchange documents, such as documents for patient referral and returning. SNOMED CT and LOINC are used as standard terminology for data items of the exchange documents. However, most of the value sets are not documented using standard terminologies. If HL7 FHIR is adopted as the technical standard for the healthcare information exchange program in the future, use of SNOMED CT will increase.

(3) Data-driven hospitals: The data-driven hospital program aims to support and promote clinical data-based research in healthcare institutions for the development of new medical technologies, such as new drugs, medical devices, and artificial intelligence. Reference sets may be developed for various research projects included in the data-driven hospital program. For example, reference sets for five types of cancers (stomach, liver, colorectal, lung, and breast cancers) will be developed in collaboration with the National Cancer Data Warehouse project.

(4) National registries: The data items included in various national registries, including the Cancer Registration and Statistics Program, the Rare Disease Registration Program, the Tuberculosis Registration Program, and the Infectious Disease Registration Program, can be mapped to SNOMED CT so that the registration data can be used for various research purposes.

(5) Public health and report: The data items included in national public health reporting systems, such as the infectious disease reporting system (e.g., COVID-19 reporting system of the Korea Disease Control and Prevention Agency) and the vaccination reporting system, can be mapped to SNOMED CT to collect high-quality data.

(6) Common data models: Among the domains of the common data model (CDM), SNOMED CT is used to map conditions, procedures, specimens, observations, devices, and measurement values. However, many of the Korean Classification of Disease codes, health insurance claim codes, and local codes cannot be mapped to SNOMED CT because the concepts are expressed only in Korea. These concepts used only in Korea can be added to the Korean extension of SNOMED CT to increase the usability of SNOMED CT.

\section{Conclusion}

SNOMED CT is a reference terminology used most widely in the world. Since Korea became the 39th member country of SNOMED International in August 2020, Korean vendors, healthcare institutions, and individual researchers can use SNOMED CT free of charge and participate in the development of SNOMED CT contents.

When SNOMED CT is used for reference terminology, clinical information can be consistently shared within a healthcare institution and between institutions; information can be organized, queried, and analyzed according to the purpose of individuals and institutions; the risk of interpreting records differently among healthcare institutions can be reduced; and data in multiple languages may be used.

Despite these advantages, there are various barriers that have to be overcome to utilize and implement SNOMED CT in clinical settings. Identified barriers include low awareness of the need for SNOMED CT, absence of SNOMED CT governance and infrastructure, insufficient SNOMED CT training and education programs, lack of support service for SNOMED CT use (e.g., translation, mapping, reference set), and lack of vendor capability to implement SNOMED CT.

Therefore, to facilitate the adoption and implementation of SNOMED CT in Korea, the infrastructure and governance for SNOMED CT management should be established. Furthermore, education and training courses should be developed and implemented for various levels and roles of the SNOMED CT users, including healthcare information experts, mapping experts, system developers, and data analysts. In addition, support service for SNOMED CT use should be facilitated by supporting translation, mapping, and the development of reference sets and clear guidelines for use and 
implementation of SNOMED CT. Moreover, SNOMED CT use cases in EHR systems and national health IT initiatives should be developed and implemented. These strategies for the adoption and implementation of SNOMED CT must be executed step by step.

\section{Conflict of Interest}

Hyeoun-Ae Park is a member of the Editorial Board of Healthcare Informatics Research; however, she did not involve in the peer review evaluation and decision process of this article. Otherwise, no potential conflict of interest relevant to this article was reported.

\section{Acknowledgments}

This research was supported by the Ministry of Health and Welfare, Republic of Korea.

\section{ORCID}

Hyeoun-Ae Park (http://orcid.org/0000-0002-3770-4998)

Hyesil Jung (http://orcid.org/0000-0002-8346-9343)

Seung-Jong Yu (http://orcid.org/0000-0003-4148-6206)

\section{References}

1. Healthcare Information and Management Systems Society. Interoperability in healthcare [Internet]. Chicago (IL): Healthcare Information and Management Systems Society Inc.; c2021 [cited at 2021 Feb 2]. Available from: https://www.himss.org/resources/interoperabilityhealthcare.

2. Cimino JJ. Desiderata for controlled medical vocabularies in the twenty-first century. Methods Inf Med 1998; 37(4-5):394-403.

3. SNOMED International. SNOMED CT Release Statistics International Edition 2021-01-31 [Internet]. London, UK: SNOMED International; c2021 [cited at $2021 \mathrm{Feb}$ 2]. Available from: https://browser.ihtsdotools.org/qa/\#/ generalReleaseStatistics.

4. SNOMED International. Data analytics with SNOMED CT [Internet]. London, United Kingdom: London, UK: SNOMED International; 2017 [cited at 2021 Feb 2]. Available from: https://confluence.ihtsdotools.org/display/DOCANLYT.

5. SNOMED International. Vendor introduction to SNOMED CT: choosing an approach to implementa- tion [Internet]. London, United Kingdom: London, UK: SNOMED International; c2021 [cited at $2021 \mathrm{Feb} 2$ ]. Available from: https://confluence.ihtsdotools.org/display/DOCVENDOR/4+Choosing+an+Approach+to+I mplementation.

6. SNOMED International. Case study: Definition of the allergies subset of SNOMED CT and incorporation into our electronic health record (EHR) [Internet]. London, UK: SNOMED International; c2021 [cited at $2021 \mathrm{Feb}$ 2]. Available from: http://www.snomed.org/snomed$\mathrm{ct} /$ case-studies/definition-of-the-allergies-subset-ofsnomed-ct.

7. SNOMED International. Case study: ON DI SNOMED CT Terminology Project [Internet]. London, UK: SNOMED International; c2021 [cited at 2021 Feb 2]. Available from: http://www.snomed.org/snomed-ct/ case-studies/on-di-snomed-ct-terminology-project.

8. SNOMED International. Case study: Ontario Microbiology Results Reporting using SNOMED CT Terminology [Internet]. London, UK: SNOMED International; c2021 [cited at $2021 \mathrm{Feb} 2$ ]. Available from: http://www. snomed.org/snomed-ct/case-studies/ontario-microbiologyresults-reporting.

9. Cambell J, Geary CR, Scott Campbell W. SNOMED CT and LOINC for computable phenotypes in Alzheimer's disease [Internet]. London, UK: SNOMED International; 2019 [cited at $2021 \mathrm{Feb} 2$ ]. Available from: https://confluence.ihtsdotools.org/pages/viewpage.action?pageId= 87042651.

10. Madani S, Giuse D, Weitkamp A, McLemore M. Augmenting NLP results by leveraging SNOMED CT relationships for identification of implantable cardiac devices from patient notes [Internet]. London, UK: SNOMED International; 2019 [cited at $2021 \mathrm{Feb} 2$ ]. Available from: https://confluence.ihtsdotools.org/display/FT/201905+ Augmenting+NLP+results+by+leveraging+SNOMED+ $\mathrm{CT}+$ relationships + for + identification + of + implantable $+\mathrm{c}$ ardiac+devices+from+patient+notes.

11. NHS Digital. Electronic Prescription Service: Phase 4 information for GP practices [Internet]. Leeds, UK: NHS Digital; 2020 [cited at $2021 \mathrm{Feb}$ 2]. Available from: https://digital.nhs.uk/services/electronic-prescriptionservice/phase-4/prescriber-information.

12. NHS Digital. Notes about the standard and summary care records [Internet]. Leeds, UK: NHS Digital; c2020 [cited 2020 Oct 05]. Available from: https://www.england.nhs.uk/ourwork/accessibleinfo/resources/scr/.

13. Parisien L, Bell C. Powering Canada's national immu- 
nization app with SNOMED CT [Internet]. Montreal, Canada: Canada Health Infoway; 2017 [cited at 2021 Feb 2]. Available from: https://confluence.ihtsdotools. org/pages/viewpage.action?pageId $=45525411$.

14. Gonzalez Bernaldo de Quiros F, Otero C, Luna D. Terminology services: standard terminologies to control health vocabulary. Yearb Med Inform 2018;27(1):22733.

15. Lam S. Barrier to implementing SNOMED CT [Inter- net]. [place unknown: publisher unknown]; [cited at $2021 \mathrm{Feb} 2$ 2]. Available from: https://slideplayer.com/ slide/12722890/.

16. Cangioi G, Chronaki C, Goeeg KR, Hojen AR, Karlsson $\mathrm{D}$, Jaulent $\mathrm{MC}$, et al. Current and future use of SNOMED CT [Internet]. Brussel, Belgium: European Commission; 2016 [cited at $2021 \mathrm{Feb} 2$ ]. Available from: https://assess-ct.eu/fileadmin/assess_ct/deliverables/ final_submissions/assess_ct_ga_643818_d1.4.pdf. 